\title{
Automated recognition of wood used in traditional Japanese sculptures by texture analysis of their low-resolution computed tomography data
}

\author{
Kayoko Kobayashi ${ }^{1} \cdot$ Masanori Akada $^{2} \cdot$ Toshiyuki Torigoe $^{3} \cdot$ Setsuo Imazu $^{2} \cdot$ \\ Junji Sugiyama ${ }^{1}$
}

Received: 3 June 2015 / Accepted: 4 August 2015/Published online: 4 September 2015

(c) The Author(s) 2015. This article is published with open access at Springerlink.com

\begin{abstract}
The identification of wood species used in the cultural artifacts is important in terms of their preservation and inheritance. However, a nondestructive method is required, and wood samples must be partly cut off in conventional methods such as microscopy. In this study, we constructed a novel system for wood identification using image recognition of X-ray computed tomography images of eight major species used in Japanese wooden sculptures. Texture analyses of the computed tomography images were carried out using the gray-level co-occurrence matrix, from which 15 textural features were calculated. The $k$-nearest-neighbor algorithm combined with cross validation was applied for classification and evaluation of the system. Input datasets with a variation in image qualities (resolution, gray level, and image size) were investigated using this novel system, and the accuracy was greater than $98 \%$ when the input images had a certain quality level. Although there are still technical problems to be overcome, progress in the development of automated identification is extremely encouraging in that such an approach has the potential to make a valuable contribution in adding scientific species notion to the artifacts; otherwise, only the literal documents are available.
\end{abstract}

Kayoko Kobayashi

kayoko_kobayashi@ rish.kyoto-u.ac.jp

1 Research Institute for Sustainable Humanosphere, Kyoto University, Gokasho, Uji, Kyoto 611-0011, Japan

2 Kyushu National Museum, Dazaifu, Fukuoka 818-0118, Japan

3 Nara National Museum, Nara, Nara 630-8213, Japan
Keywords Wood identification - Pattern recognition · Texture analysis · Gray-level co-occurrence matrix · $\mathrm{X}$-ray computer tomography

\section{Introduction}

Wood identification has been providing useful information of the origins of heritages and sometimes provided a new perspective. Although it requires expertise in wood anatomy, the technique has increasingly been used by curators and archeologists owing to the list of anatomical features published by the IAWA Comittee [1, 2]. Commonly, wood samples have to be observed from three orthogonal directions, namely, the transverse, radial, and tangential directions, to obtain the appropriate information of their anatomical features. This method becomes routine after training and experience, but it is not applicable to culturally important wood works or artifacts, where only nondestructive methods are allowed to be used.

Spectroscopy is one example of a nondestructive and noninvasive method, and several attempts to identify wood have been successfully reported [3-5]. The prediction accuracy can be improved by the quality of the spectral database, but is not sufficient for the classification of a wide range of wood species. It is more efficient if the target species for classification are limited. Another nondestructive technique is X-ray computed tomography (CT). One example is the use of synchrotron radiation X-ray microtomography, where the three-dimensional (3D) wood structure has been reconstructed at a resolution of $0.5 \mu \mathrm{m}$ [6]. In this technique, wood identification based on the IAWA list [1,2] is possible. However, the sample size and machine time are limited; therefore, the measurement is limited to selected important materials. 
Kyushu National Museum was the fourth national museum in Japan, and it is devoted to the scientific investigation of artwork. Since the installation of a largescale X-ray CT instrument, nearly 2000 wooden artifacts have been inspected. Unfortunately, the images were never considered as resources for analyzing wood properties. This is because the resolution of the image was too low to apply conventional wood identification that relies on the visual inspection of microscopic anatomical features.

For the last several decades, image recognition technology, such as an automated face-recognition system and fingerprint authentication, has been significantly developed. As a method for the extraction of image information, which is one of the important processes in image recognition, a texture analysis has been utilized in a variety of fields such as remote sensing and medical imaging. Several authors have also reported attempts of the application of these techniques to wood identification [7-19], which are particularly active in tropical areas. Tropical timber is an important biological and economical resource in the developing world; thus, wood identification is demanded at trading locations to circulate proper wood in the market as well as to keep illegally logged timber under observation. Under such circumstances, an automated wood identification system is highly demanded because the development of human resources is important, but it is not straightforward to train people to have in-depth anatomical knowledge and experience to cover the large diversity of tropical hardwood species. Many studies have mostly used optical micrographs $[10,11,14,19]$ or stereograms [7-9, 12, 13, 15-18], whose resolution was a several micrometers at worst. Under such conditions, the arrangements of vessels and axial parenchyma were clearly recorded and used as image information. Several approaches for the extraction of image information were tested, such as the segmentation of specific anatomical features [10, 11], the gray-level cooccurrence matrix (GLCM) [7-9], Gabor filtering [16], Gabor filtering followed by the GLCM [12], the Coiflet discrete wavelet transform [18], the extended higher local order autocorrelation [15], and local binary patterns [14]. Combinations of multiple extractors were also tested for further improvement in the systems [13, 17, 19].

Inspired by the above-mentioned development of automated recognition systems, the computer-aided recognition of low-resolution CT images recorded at Kyushu National Museum seemed to have a potential to be uncovered. Although the resolution of the original CT images does not allow for visualization of individual anatomical features, the density gradient and fluctuation that are specific to the species are certainly recorded.

Therefore, the eight most frequently used wood species in Japanese sculptures were selected and analyzed in this study. As for the feature extraction procedure, the most simple and reputable technique, the GLCM that considers the spatial gray-level distribution [20], was considered to be the best as the first choice. The detailed procedures and the applicability of the automated wood identification technique will be described.

\section{Methods}

\section{Wood samples}

Ten types of wood blocks were selected by Mr. Yano Ken'ichiro at the Tokyo University of the Arts, Institute of Ancient Art Research, Nara from critical/practical viewpoints as a professional sculptor. They are Cinnamomum camphora (Cc), Cercidiphyllum japonicum (Ce), Cryptomeria japonica* (Cj), Chamaecyparis obtusa* (Co), Magnolia obovata (Mo), Pawlounia tomentosa (Pt), Torreya nucifera $(\mathrm{Tn})$, and Zelkova serrata $(\mathrm{Zs})$. Species with asterisk have two variations - one from a plantation forest $(\mathrm{Cj} 1, \mathrm{Co} 1)$ and the other from a natural forest $(\mathrm{Cj} 2, \mathrm{Co} 2)$.

\section{X-ray computed tomography}

X-ray CT experiments of the wood samples were performed using the large-scale X-ray CT instrument (Y.CT Modular, YXLON International GmbH, Germany) at the Kyushu National Museum. Al-filtered X-rays were generated at $225 \mathrm{kV}$ and $30 \mathrm{~mA}$. A total of 1080 projections were recorded on a flat-panel detector and reconstructed by employing the filtered back-projection procedure. The sample-source distance was set to achieve a voxel resolution of $0.05 \mathrm{~mm}$.

\section{Initial image dataset}

From the reconstructed 3D dataset of the wood blocks, two-dimensional (2D) images were precisely cropped in the direction that provides a transverse section using volume graphics software (VGStudio MAX 2.1, Volume Graphics GmbH, Germany). The images have dimensions of 300 pixels $\times 300$ pixels that correspond to $1.5 \mathrm{~cm} \times 1.5 \mathrm{~cm}$ in actual size and a 256-level gray scale. Forty images were randomly collected for each wood block, and a total of 400 images in the TIFF file format were used as an initial dataset.

\section{Computational approaches}

An outline of the analysis procedure is shown in Fig. 1. From the initial dataset, datasets with a variety of qualities were created, and each dataset was then input into the recognition system. The system consists of two steps: 
Fig. 1 Schematic diagram of the analysis procedure

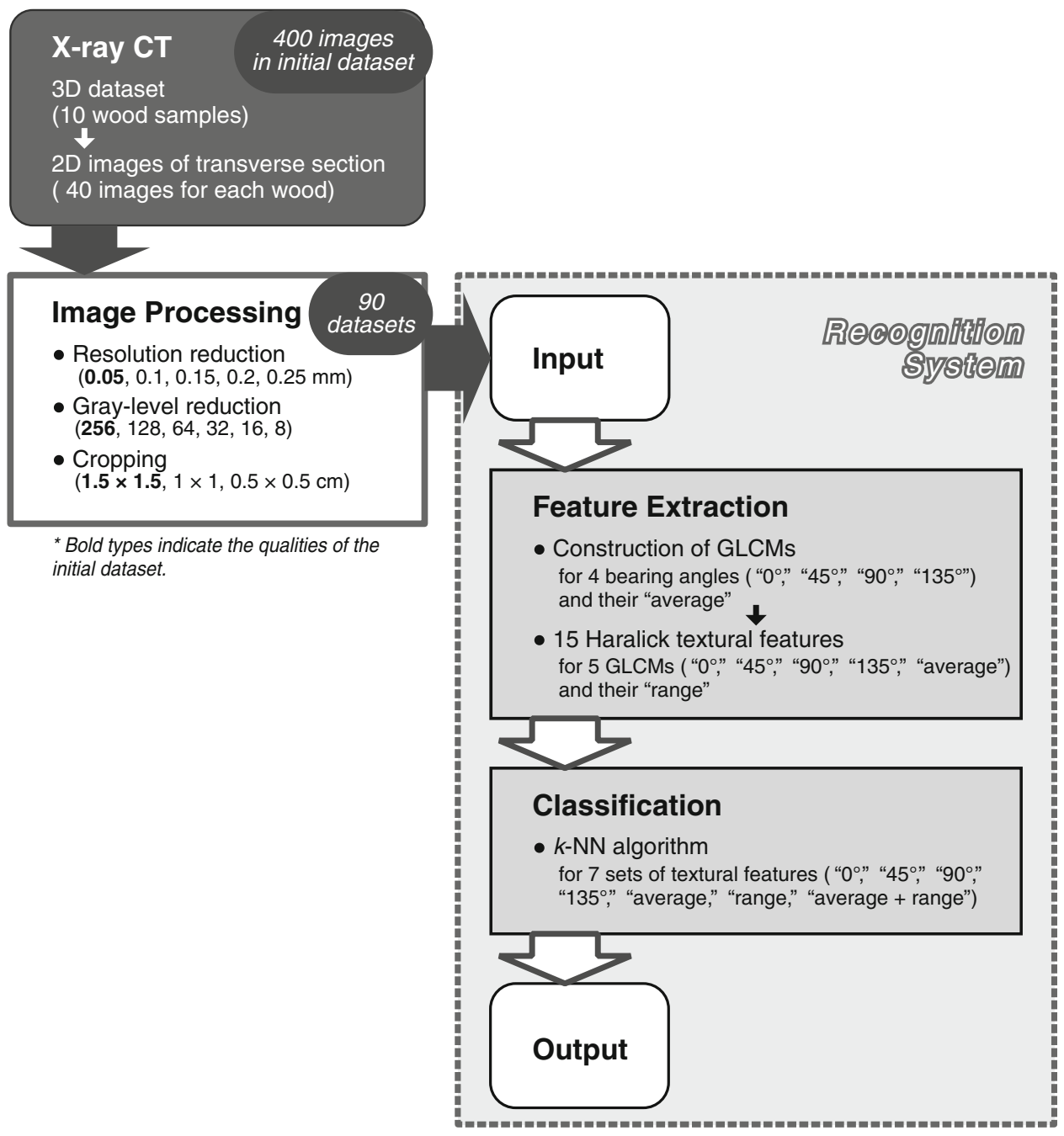

feature extraction and classification. Statistical processing of all of the following images was carried out using $\mathrm{R}$ version 3.1.1 [21] and its packages.

\section{Preprocessing images}

In addition to the initial dataset, a number of datasets was created by reductions in the resolution, the number of gray levels, and the image size. The resolution and image size, which were originally $0.05 \mathrm{~mm}$ and $1.5 \mathrm{~cm} \times 1.5 \mathrm{~cm}$, respectively, were changed using a function in the "biOps" package [22]. The resolution was reduced to $0.1,0.15,0.2$, and $0.25 \mathrm{~mm}$, and the image size was reduced to $1 \mathrm{~cm} \times 1 \mathrm{~cm}$ and $0.5 \mathrm{~cm} \times 0.5 \mathrm{~cm}$. The number of gray levels, which was initially $256\left(=2^{8}\right)$, was reduced, and images with $2^{n}$ ( $n=3-7)$ gray levels were generated. In total, 90 datasets with five resolutions, six different numbers of gray levels, and three image sizes were used for further analysis.

\section{Feature extraction}

The GLCM from an image with $2^{n}$ gray levels is a matrix with dimensions of $2^{n} \times 2^{n}$. The matrix $P(i, j)$ represents the probability of occurrence of the pair of gray levels $i$ and $j$ at a separation distance $d$. In our study, $d$ was fixed to 1 , and the GLCMs were calculated from four directions, which are horizontal (" $0^{\circ}$ "), vertical (" $90^{\circ}$ "), and diagonal (" $45^{\circ}$ " and " $135^{\circ}$ "). In addition to the four GLCMs, one new matrix was added, which is the "average" of those of the four directions.

A total of 15 textural features were extracted from each GLCM, including the 13 textural features proposed by Haralick et al. [20] and the two textural features added by Albregtsen [23], which are given as follows: 
Angular second moment (ASM) $f_{1}=\sum_{i=0}^{G-1} \sum_{j=0}^{G-1}\left\{P(i, j)^{2}\right\}$

Contrast $f_{2}=\sum_{i=0}^{G-1} \sum_{j=0}^{G-1}(i-j)^{2} P(i, j)$

$\operatorname{IDM} f_{3}=\sum_{i=0}^{G-1} \sum_{j=0}^{G-1} \frac{1}{1+(i-j)^{2}} P(i, j)$

Entropy $f_{4}=-\sum_{i=0}^{G-1} \sum_{j=0}^{G-1} P(i, j) \log P(i, j)$

Correlation $f_{5}=-\sum_{i=0}^{G-1} \sum_{j=0}^{G-1} \frac{\left(i-\mu_{x}\right)\left(j-\mu_{y}\right)}{\sqrt{\sigma_{x} \sigma_{y}}} P(i, j)$

Variance $f_{6}=\sum_{i=0}^{G-1} \sum_{j=0}^{G-1}(i-\mu)^{2} P(i, j)$

Sum Average $f_{7}=\sum_{i=0}^{2 G-2} i P_{x+y}(i)$

Sum Entropy $f_{8}=-\sum_{i=0}^{2 G-2} P_{x+y}(i) \log \left\{P_{x+y}(i)\right\}$

Difference Entropy $f_{9}=-\sum_{i=0}^{G-1} P_{x-y}(i) \log \left\{P_{x-y}(i)\right\}$

Difference Variance $f_{10}=\sum_{i=0}^{G-1}\left(i-f_{9}\right)^{2} P_{x-y}(i)$

Sum Variance $f_{11}=\sum_{i=0}^{2 G-2}\left(i-f_{8}\right)^{2} P_{x+y}(i)$

$f_{12}=\frac{H X Y 1-f_{4}}{\max \{H X, H Y\}}$

$f_{13}=\sqrt{1-\exp \left[-2\left(H X Y 2-f_{4}\right)\right]}$

Shade $f_{14}=\sum_{i=0}^{G-1} \sum_{j=0}^{G-1}\left\{i+j-\mu_{x}-\mu_{y}\right\}^{3} P(i, j)$

Prominence $f_{15}=\sum_{i=0}^{G-1} \sum_{j=0}^{G-1}\left\{i+j-\mu_{x}-\mu_{y}\right\}^{4} P(i, j)$

where

$P(i, j)$ is the $(i, j)$ thelement of a GLCM, and

$G$ is the number of gray levels.

$$
\begin{aligned}
& P_{x}(i)=\sum_{i=0}^{G-1} P(i, j), P_{y}(j)=\sum_{i=0}^{G-1} P(i, j), \\
& \mu_{x}=\sum_{i=0}^{G-1} i P_{x}(i), \quad \mu_{y}=\sum_{j=0}^{G-1} j P_{y}(j), \quad \mu=\frac{\mu_{x}+\mu_{y}}{2},
\end{aligned}
$$

$$
\begin{aligned}
& \sigma_{x}^{2}=\sum_{i=0}^{G-1}\left(i-\mu_{x}\right)^{2} P_{x}(i), \quad \sigma_{y}^{2}=\sum_{i=0}^{G-1}\left(j-\mu_{y}\right)^{2} P_{y}(j), \\
& P_{x+y}(k)=\sum_{i=0}^{G-1} \sum_{j=0}^{G-1} P(i, j), \\
& P_{x-y}(l)=\sum_{i=0}^{G-1} \sum_{j=0}^{G-1} P(i, j)(i+j=k,|i-j|=l) \\
& H X=-\sum_{i=0}^{G-1} P_{x}(i) \log P_{x}(i), \quad H Y=-\sum_{j=0}^{G-1} P_{y}(j) \log P_{y}(j), \\
& H X Y 1=-\sum_{i=0}^{G-1} \sum_{j=0}^{G-1} P(i, j) \log \left\{P_{x}(i) P_{y}(j)\right\} \\
& H X Y 2=-\sum_{i=0}^{G-1} \sum_{j=0}^{G-1} P(i) P(j) \log \left\{P_{x}(i) P_{y}(j)\right\} .
\end{aligned}
$$

In addition to the five sets of 15 features extracted from the GLCMs, one more set was added, which is the "range" of the textural features of the GLCM in the four directions.

\section{Verification and classification}

A principal component analysis (PCA) using the "stats" package [21] was carried out to evaluate the textural features suitable for the recognition procedure. From one image, six sets of 15 features were already obtained. Then, one more set was included, which is a linear combination of "average + range" [20], consisting of 30 textural features. A total of seven sets of features, " $0^{\circ}$ ", "90", "45 ", "135 ", "average", "range" and "average + range" were tested and evaluated in terms of species-specific clustering.

The $k$-nearest-neighbor $(k-\mathrm{NN})$ algorithm was used for discrimination combined with leave-one-out cross validation (LOOCV) using the "class" package [24]. In the LOOCV method, one object is drawn from the entire dataset as a test set, which is classified according to a model built by the rest of the objects. On the basis of the results obtained by repeating this process for all objects, the performance of the discrimination model is estimated.

The rate of correctly classified objects was calculated when the value of $k$ is an odd number, and the capability of the recognition was evaluated by the average of the accuracy with $k=1,3,5,7$, and 9 . In total, 90 image datasets with five resolutions, six different numbers of gray levels, and three image sizes were subjected to the classification procedure using seven sets of GLCM-related textural features. 


\section{Results and discussion}

\section{Extraction of features from CT images}

Figure 2 shows the representative CT images of transverse sections without any image processing, where a darker area corresponds to an area of lower density. The images were cropped so that the annual rings are arranged in the vertical direction, although some images from Cryptomeria japonica from the plantation forest $(\mathrm{Cj} 1)$ were difficult to arrange in this manner because its wood block had smaller annual rings in diameter. The microscopic anatomical features were not well observed in these images as expected, but they obviously showed some differences among the species. In particular, features related to the annual rings, such as their widths and the gradient from a darker area to a lighter area that corresponds to earlywood to latewood in an annual ring, were readily recognized. The annual ring boundaries in softwood such as $\mathrm{Cj}$ and $\mathrm{Co}$, for instance, were quite sharp, whereas diffuse-porous hardwood such as Ce and Mo exhibited blurred images. In addition, large pores were also observed as dark dots or lines in ring-porous hardwoods, $\mathrm{Pt}$ and $\mathrm{Zs}$, and diffuseporous hardwoods with large vessels, Cc.

These CT images were subsequently converted into GLCMs, which represent the probabilities of each combination of gray levels of neighboring pairs occurring in an image. Figure 3 shows the GLCMs obtained from some of the images in Fig. 2 after a reduction in the number of gray levels to 64; thus, the GLCMs consist of 64 columns and 64 rows. Comparing the GLCMs of Zs among the four bearing angles (Fig. 3a), the elements in the GLCM of " $90^{\circ}$," which corresponded to the direction along the annual rings, were more concentrated along the diagonal than those of
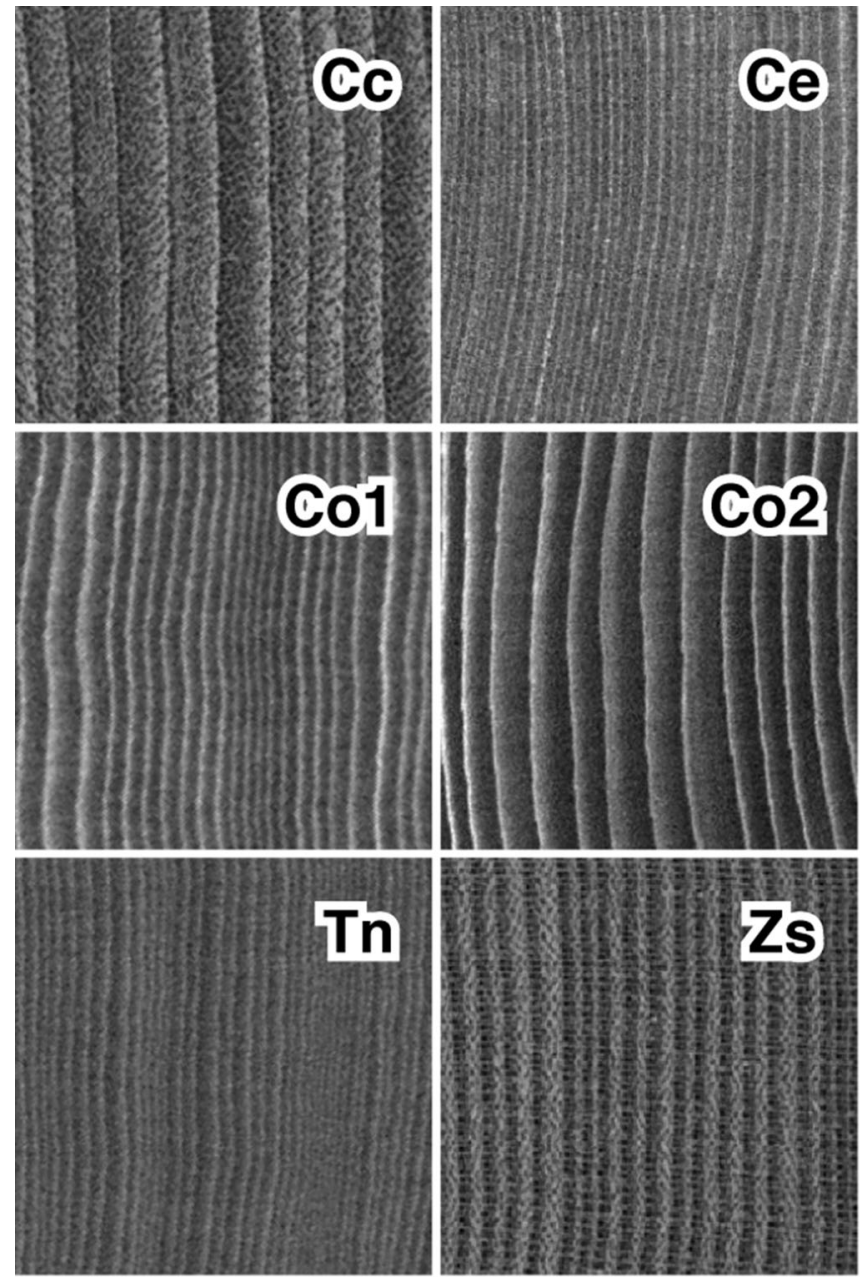

Fig. 2 Typical cross-sectional 2D images from CT datasets obtained from 10 types of woods, with arrows indicating the directions of the bearing angles for the construction of GLCMs. Cc, Cinnamomum camphora; $\mathrm{Ce}$, Cercidiphyllum japonica; $\mathrm{Cj} 1$, Cryptomeria japonica (from a plantation forest); $\mathrm{Cj} 2$, Cryptomeria japonica (from a natural
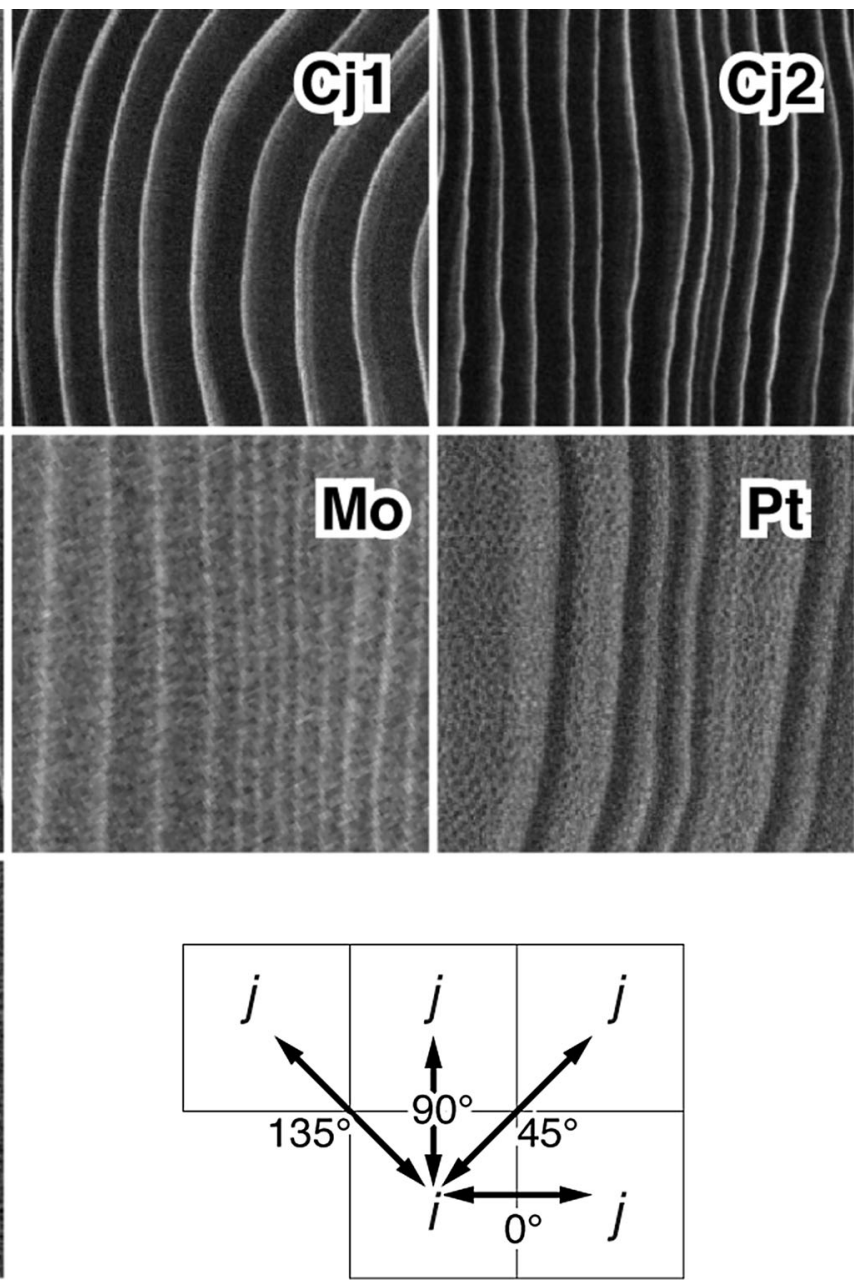

forest); Co1, Chamaecyparis obtusa (from a plantation forest); $\mathrm{Co} 2$, Chamaecyparis obtusa (from a natural forest); Mo, Magnolia obovata; Pt, Paulownia tomentosa; Tn, Torreya nucifera; and Zs, Zelkova serrata. The images have a resolution of $0.05 \mathrm{~mm}, 256$ gray levels, and an actual size of $1.5 \mathrm{~cm} \times 1.5 \mathrm{~cm}$ 

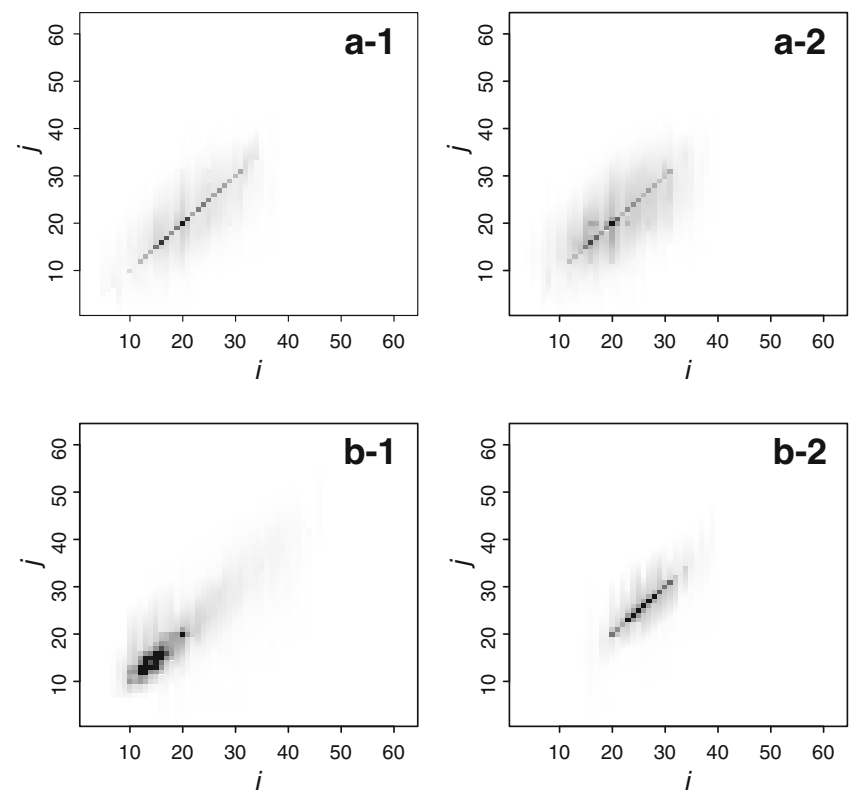

Fig. 3 Gray-level co-occurrence matrices (GLCMs) calculated from some of the images in Fig. 2 with a reduction of the number of gray levels to 64. Elements with higher values are indicated by darker colors. a Comparison of the GLCMs obtained from Zs with different

the other directions, indicating that more neighboring pixels have the same or nearly the same gray level. On the other hand, the elements were slightly dispersed from the diagonal in the GLCM of " 0 "," which resulted from the transition of gray levels across the annual rings. The GLCMs of " $45^{\circ}$ " and " $135^{\circ}$," consisting of neighboring pairs in the direction diagonally across the annual rings, exhibited more dispersed patterns.

The GLCMs of Cj2, Mo, and Zs obtained with the "average" among the four bearing angles are shown in Fig. 3b. In the GLCM of Cj2, many elements gathered at the left lower side, but a small quantity of elements also spread to the right upper side for a wide range (Fig. 3b-1). This is because the image of $\mathrm{Cj} 2$ mainly consists of large dark areas corresponding to earlywood, but they gradually transition to small light areas corresponding to latewood. From the blurred image of Mo, on the other hand, the GLCM consisted of fewer elements, and they were concentrated on the diagonal (Fig. 3b-2). In the GLCM of Zs (Fig. 3b-3), there are also many elements along the diagonal, and some of them are dispersed from the diagonal. Some neighboring pairs in the image of Zs have large differences in gray levels due to the large pores.

From the GLCMs obtained from the images, 15 textural features were calculated. Here, the meanings of the features are specifically explained using three of them calculated from the GLCMs shown in Fig. $3 b$ as concrete examples (Fig. 4). ASM is the sum of the squares of all elements, as expressed by Eq. 1; thus, a GLCM consisting of fewer
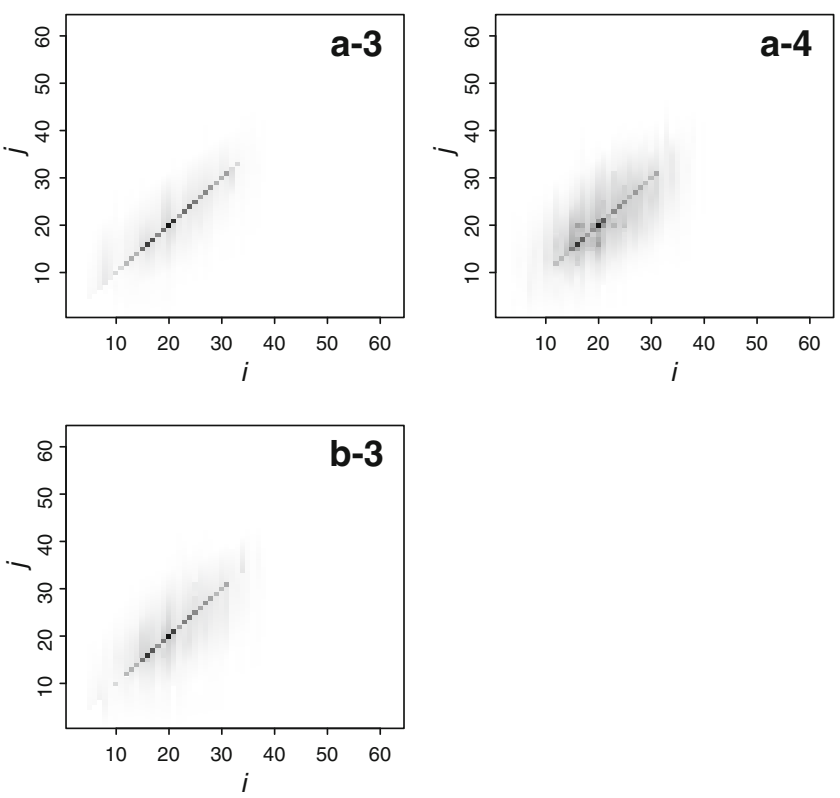

bearing angles of " 0 ", " $45^{\circ} "$, " $90^{\circ}$ " and " $135^{\circ}$ ". b Comparison of GLCMs obtained from different wood species: Co2, Mo, and Zs. The parameter used for the calculation is "average"

elements makes ASM larger, representing a measure of homogeneity. Comparing the values of ASM among the three wood species, the value for Mo was much greater than those for $\mathrm{Co} 2$ and $\mathrm{Zs}$, which is consistent with the visual appearances of the images. Because contrast, which becomes larger if there are more elements in the GLCM far from the diagonal of $i=j$ (Eq. 2), represents the amount of the local density variation, $\mathrm{Co} 2$ and $\mathrm{Zs}$ exhibited larger values. Variance is the degree of variability from the average of all elements (Eq. 3), resulting in the largest value for $\mathrm{Co} 2$ among the three because the difference in gray levels is extreme in its image.

\section{Classification and evaluation of the recognition system}

Before classification using the extracted textural features, they were analyzed by a PCA for testing the clustering of objects and the dispersion of the textural features in the feature space. Figure 5 shows examples of the results of the PCA, which were obtained using the features calculated from "average," "range," and "average + range" of images with 64 gray levels. In most cases, the 15 textural features pointed in diverse directions in the score plot, as shown in Fig. 5a, indicating that every feature has meaningful information for discrimination. As a result, the objects were well dispersed and clustered for each class. In contrast, in some cases shown in Fig. 5b, many textural features exhibited similar directions, and several clusters 
Fig. 4 Comparison of three selected textural features for Co2, Mo, and Zs calculated from the GLCMs in Fig. 3b
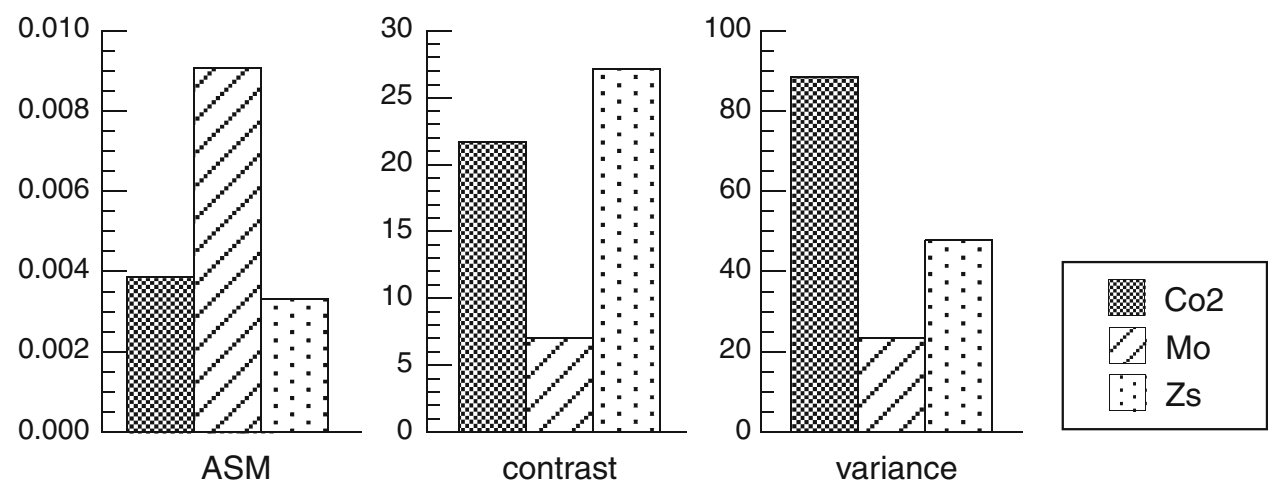

variance
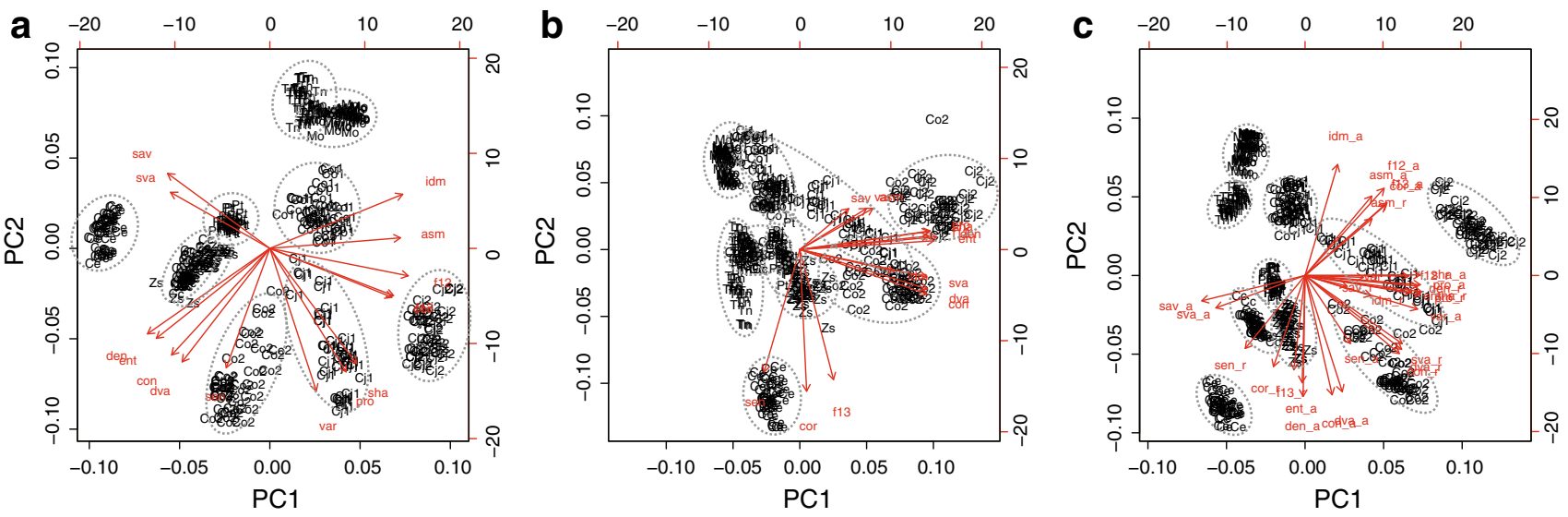

Fig. 5 Biplots of the first and second principal components (PC1 and PC2) with parameters of a "average", b "range", and c "aver-

image size are $0.05 \mathrm{~mm}, 64$, and $1.5 \times 1.5 \mathrm{~cm}$, respectively. The cumulative contribution ratios of $\mathrm{PC} 1$ and $\mathrm{PC} 2$ in (a), (b), and (c) are age + range" when the resolution, the number of gray levels, and the

83,70 , and $72 \%$, respectively
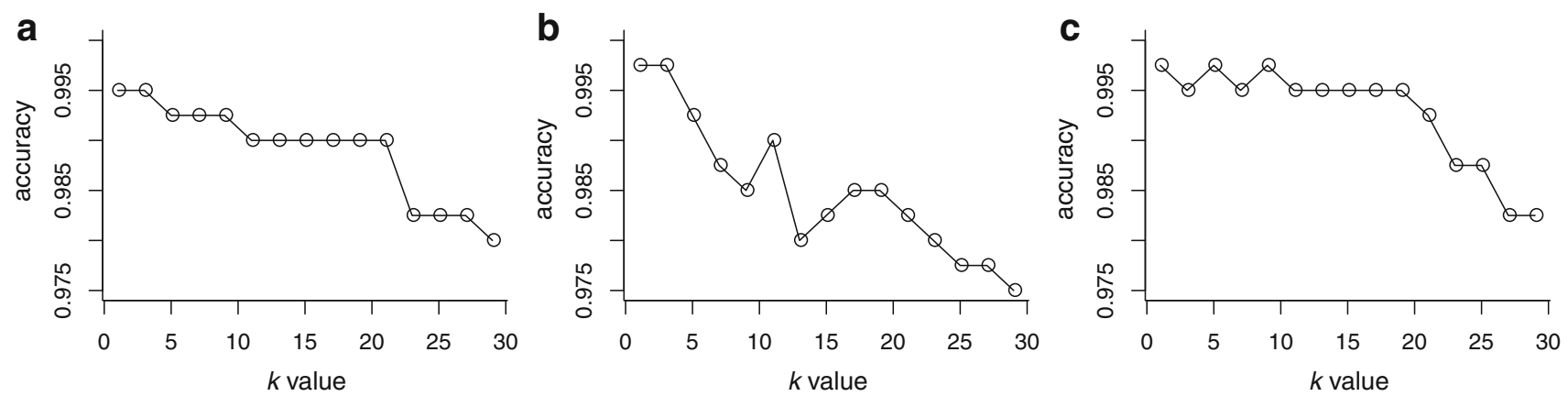

Fig. 6 Changes in the accuracy for $k$-nearest neighbor classification with the parameters of a "average", b "range", and c "average + range" when the resolution, the number of gray levels, and the image size are $0.05 \mathrm{~mm}, 64$, and $1.5 \times 1.5 \mathrm{~cm}$, respectively

for each class overlapped with each other. Using the combined 30 features of "average + range" (Fig. 5c), the objects seemed to be discriminated as well as or better than the case in which only the 15 features of "average" were used, even though some of the features pointed in similar directions.

The $k$-NN algorithm was adopted for classification, and the accuracy of the recognition system was estimated by LOOCV for each value of $k$. Figure 6 shows the changes in the accuracy depending on $k$ when using the same dataset and parameters as in Fig. 5. The accuracy remained almost constant with the increase in $k$ when "average" and "average + range" were used (Fig. 6a, c), whereas it gradually decreased in the case of "range" (Fig. 6b). This is because an object is misclassified into the wrong class with a higher probability with increasing $k$ if there is an object located between its true cluster and the other ones. These results were consistent with the PCA analysis, where 
Table 1 Optimum parameters for each condition

\begin{tabular}{|c|c|c|c|c|c|c|c|c|c|c|c|c|c|c|c|}
\hline \multirow{2}{*}{$\begin{array}{l}\text { Image size } \\
\left(\mathrm{cm}^{2}\right) \\
\text { Resolution } \\
(\mathrm{mm} / \text { pixel })\end{array}$} & \multicolumn{5}{|l|}{$1.5 \times 1.5$} & \multicolumn{5}{|l|}{$1 \times 1$} & \multicolumn{5}{|c|}{$0.5 \times 0.5$} \\
\hline & 0.05 & 0.1 & 0.15 & 0.2 & 0.25 & 0.05 & 0.1 & 0.15 & 0.2 & 0.25 & 0.05 & 0.1 & 0.15 & 0.2 & 0.25 \\
\hline \multicolumn{16}{|l|}{ Gray level } \\
\hline 8 & $a+r$ & $0^{\circ}$ & $a+r$ & $0^{\circ}$ & $0^{\circ}$ & $a+r$ & $0^{\circ}$ & $a+r$ & $0^{\circ}$ & $0^{\circ}$ & $a+r$ & $a+r$ & $a+r$ & $a+r$ & $0^{\circ}$ \\
\hline 16 & $a+r$ & ave & $a+r$ & $0^{\circ}$ & $0^{\circ}$ & $a+r$ & $0^{\circ}$ & $a+r$ & $0^{\circ}$ & ave & $a+r$ & $0^{\circ}$ & $a+r$ & $0^{\circ}$ & $0^{\circ}$ \\
\hline 32 & $a+r$ & ave & $a+r$ & $0^{\circ}$ & $0^{\circ}$ & $a+r$ & $0^{\circ}$ & $0^{\circ}$ & $0^{\circ}$ & $0^{\circ}$ & $a+r$ & $0^{\circ}$ & $a+r$ & $0^{\circ}$ & $0^{\circ}$ \\
\hline 64 & $\begin{array}{l}0^{\circ}, 90^{\circ} \\
\mathrm{a}+\mathrm{r}\end{array}$ & $90^{\circ}$ & $a+r$ & $a+r$ & ave & $90^{\circ}$ & $a+r$ & $a+r$ & $0^{\circ}$ & $0^{\circ}$ & $a+r$ & $0^{\circ}$ & $a+r$ & $0^{\circ}$ & $0^{\circ}$ \\
\hline 128 & $90^{\circ}$ & $90^{\circ}$ & $a+r$ & $0^{\circ}$ & ave & $90^{\circ}$ & $a+r$ & $a+r$ & $0^{\circ}$ & $0^{\circ}$ & ave & $0^{\circ}$ & $a+r$ & $0^{\circ}$ & ave \\
\hline 256 & $0^{\circ}, 90^{\circ}$, ave & $90^{\circ}$, ave & $a+r$ & $0^{\circ}$ & ave & $a+r$ & $a+r$ & $a+r$ & $0^{\circ}$ & $0^{\circ}$ & ave & $a+r$ & $0^{\circ}$ & $0^{\circ}$ & $0^{\circ}$, ave, $a+r$ \\
\hline
\end{tabular}

ave "average", $a+r$ "average + range"

"average" and "average + range" exhibited good clustering compared to "range" (Fig. 5). The maximum value at a certain value of $k$, therefore, was not appropriate to evaluate the recognition system because the values would have small differences when comparing the systems with the three different parameters shown in Fig. 6. Alternatively, the average values with $k=1,3,5,7$, and 9, which include the information of the degreasing level from the maximum value, were used in this study as the predicted accuracy of the recognition system.

\section{Validation of the output results}

\section{Selection of the optimum parameters}

The predicted accuracies were calculated for the seven parameters, and the parameter that provided the highest predicted accuracy was examined for all of the input datasets (Table 1). For input images with a high resolution and a large number of gray levels, such as a resolution above $0.1 \mathrm{~mm}$ and 64 gray levels, there were no significant differences among the values obtained using the parameters of " $0^{\circ} "$, " $90^{\circ} "$, "average" and "average + range". The information integrated from all directions is commonly used for image recognition of isotropic objects, whereas wood is an anisotropic object with three characteristic orthogonal directions. For this reason, the data obtained using the parameters of " 0 " " and " $90^{\circ}$ ", which correspond to the characteristic directions in the radial and tangential directions, respectively, captured the features of the wood structure well, which resulted a high performance and the use of "average" and "average + range." With decreases in the resolution and the numbers of gray level, however, the variability in the predicted accuracy depending on the parameters became larger. With a slight decrease in the image qualities, "average + range" tended to give the best prediction because it included a significant amount of information from all directions and the degree of anisotropy in the images. When the resolution became even lower, such as 0.2 and $0.25 \mathrm{~mm}$, the optimum parameter was " 0 " in most cases. At these levels of resolution, fine differences in the gray level that originate from the different types of the cells could not be recognized, and only the information from the direction across the annual rings, such as the degree of the gradient from earlywood to latewood, would be meaningful. "Average + range" has an advantage in that it is applicable to images with any arrangement, whereas the images used in this study were cropped so that the annual rings were arranged in the same direction, which would be difficult for objects with limited wooden areas. On the other hand, " 0 " " would be better if the data could be obtained with a low resolution but from a large area. Therefore, the textural features of "average + range" or " 0 " should be used according to the situation for the construction of the discriminant model.

\section{Required qualities of the input data}

The predicted accuracies obtained using the parameters "average + range" and " 0 " are shown in Figs. 7 and 8, respectively. In either case, the predicted accuracy was greater than 0.99 for the initial dataset with the best quality, indicating almost all images were classified into their true classes. With a reduction in the resolution, the number of gray levels, or the image size, the predicted accuracy basically decreased. However, the number of gray levels had a lower effect on the accuracy, except for eight gray levels. The number of gray levels could be reduced to 16 or 32, which will be a great advantage to the system for significant reductions in the calculation time and database capacity. The resolution of $0.1 \mathrm{~mm}$ resulted in a high predicted accuracy as did $0.05 \mathrm{~mm}$, but the accuracy 

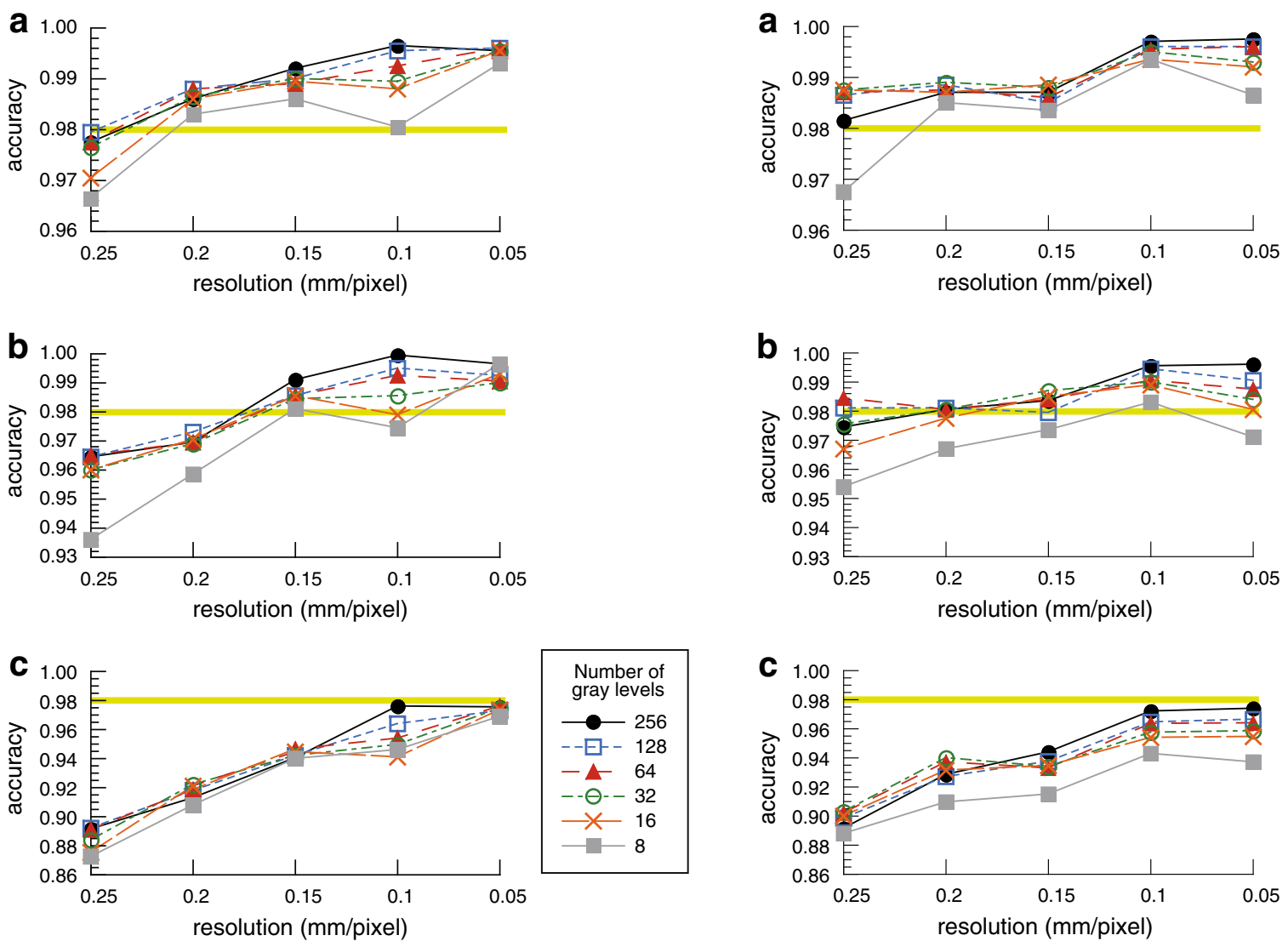

Fig. 7 Predicted accuracies for all resolutions and numbers of gray levels with images sizes of a $1.5 \times 1.5 \mathrm{~cm}, \mathbf{b} 1 \times 1 \mathrm{~cm}$, and c $0.5 \times 0.5 \mathrm{~cm}$ using the parameter "average + range". The bold lines are drawn at $98 \%$ as an indication of the required accuracy

gradually decreased at lower resolutions, particularly for "average + range." The image size was the most important factor for obtaining a high accuracy. The predicted accuracy decreased greatly with a decrease from $1 \mathrm{~cm} \times 1 \mathrm{~cm}$ to $0.5 \mathrm{~cm} \times 0.5 \mathrm{~cm}$.

Assuming that the required accuracy of the system for practical application is greater than $98 \%$, any conditions with the image size of $0.5 \mathrm{~cm} \times 0.5 \mathrm{~cm}$ could not achieve this accuracy. If images larger than $1 \mathrm{~cm} \times 1 \mathrm{~cm}$ were obtained, the resolution of $0.15 \mathrm{~mm}$ would be sufficient, and even $0.25 \mathrm{~mm}$ would be possible when the parameter " 0 " " is used. Currently, the maximum resolution that could be obtained from large objects such as human-sized sculptures is $0.2 \mathrm{~mm}$ because the sample-source distance defined the resolution; thus, the results indicated that the system has the potential for application to such large sculptures if we could ensure a sufficient size of the wooden part, such as $1.5 \mathrm{~cm} \times 1.5 \mathrm{~cm}$. In addition, the system could also be applicable to objects with small wooden parts, such as $1 \mathrm{~cm} \times 1 \mathrm{~cm}$, e.g., wooden frames used in dry lacquer.

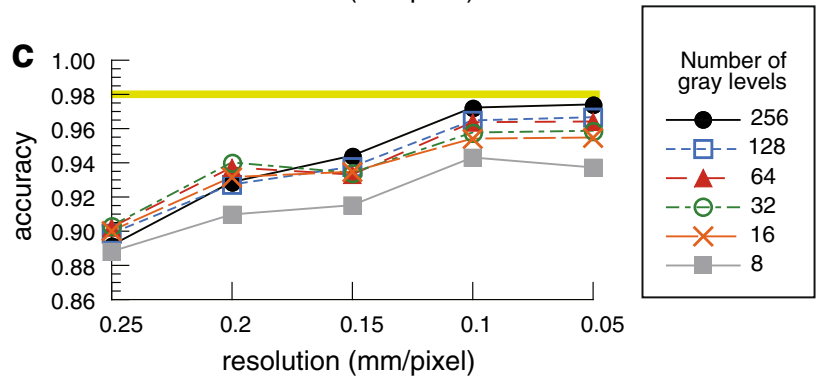

Fig. 8 Predicted accuracies for all resolutions and numbers of gray levels with images sizes of a $1.5 \times 1.5 \mathrm{~cm}, \mathbf{b} 1 \times 1 \mathrm{~cm}$, and c $0.5 \times 0.5 \mathrm{~cm}$ using the parameter " 0 " ". The bold lines are drawn at $98 \%$ as an indication of the required accuracy

\section{Misclassified objects}

Although the wood samples were classified into their true classes with a high accuracy, there were still some misclassifications. For a resolution of $0.05 \mathrm{~mm}$, the most misclassified object was $\mathrm{Pt}$, which was classified into $\mathrm{Zs}$ (Table 2). The textures of these two wood species were similar because both are ring-porous hardwood, where large vessels are arranged in the earlywood, which were visualized as dark bands in the CT image, and small vessels with parenchyma cells are arranged in a line in the latewood, which appeared as black streaks in the light bands in the CT images (Fig. 2, Pt, Zs).

In contrast, with a lower resolution of $0.1 \mathrm{~mm}$, the misclassified objects completely changed (Table 2). In many cases, Zs was misclassified as $\mathrm{Cc}$, whereas there was no misclassification of $\mathrm{Pt}$ as $\mathrm{Zs}$. Cc is a diffuse-porous hardwood but is also described as a semi-ring-porous hardwood, where there are large vessels in the earlywood that become slightly smaller in the latewood. However, these vessels in $\mathrm{Cc}$ are randomly distributed, which is in 
Table 2 Classification table for five different numbers of gray levels $(16,32,64,128$, and $256)$, five $k$ values $(1,3,5,7$, and 9), and two parameters (" 0 " " and "average + range") for an image size of $3 \times 3 \mathrm{~cm}$

\begin{tabular}{|c|c|c|c|c|c|c|c|c|c|c|}
\hline & \multicolumn{10}{|c|}{ True class } \\
\hline & $\mathrm{Cc}$ & $\mathrm{Ce}$ & $\mathrm{Cj} 1$ & $\mathrm{Cj} 2$ & Co1 & $\mathrm{Co} 2$ & Mo & $\mathrm{Pt}$ & $\operatorname{Tn}$ & Zs \\
\hline \multicolumn{11}{|c|}{ Resolution of $0.05 \mathrm{~mm}$} \\
\hline \multicolumn{11}{|c|}{ Classified class $(\%)$} \\
\hline $\mathrm{Cc}$ & 100 & 0 & 0 & 0 & 0 & 0.10 & 0 & 0 & 0 & 0.50 \\
\hline $\mathrm{Ce}$ & 0 & 100 & 0 & 0 & 0 & 0 & 0 & 0 & 0 & 0 \\
\hline $\mathrm{Cj} 1$ & 0 & 0 & 99.35 & 0 & 0 & 0 & 0 & 0 & 0 & 0 \\
\hline $\mathrm{Cj} 2$ & 0 & 0 & 0 & 100 & 0 & 0.50 & 0 & 0 & 0 & 0 \\
\hline $\mathrm{Co} 1$ & 0 & 0 & 0.65 & 0 & 100 & 0 & 0 & 0 & 0 & 0 \\
\hline $\mathrm{Co} 2$ & 0 & 0 & 0 & 0 & 0 & 99.30 & 0 & 0 & 0 & 0 \\
\hline Mo & 0 & 0 & 0 & 0 & 0 & 0 & 100 & 0 & 0 & 0 \\
\hline $\mathrm{Pt}$ & 0 & 0 & 0 & 0 & 0 & 0 & 0 & 97.50 & 0 & 0.30 \\
\hline $\mathrm{Tn}$ & 0 & 0 & 0 & 0 & 0 & 0 & 0 & 0 & 100 & 0 \\
\hline Zs & 0 & 0 & 0 & 0 & 0 & 0.10 & 0 & 2.50 & 0 & 99.20 \\
\hline \multicolumn{11}{|c|}{ Resolution of $0.1 \mathrm{~mm}$} \\
\hline \multicolumn{11}{|c|}{ Classified class (\%) } \\
\hline $\mathrm{Cc}$ & 99.40 & 0 & 0 & 0 & 0 & 0.90 & 0 & 0.10 & 0 & 4.30 \\
\hline $\mathrm{Ce}$ & 0 & 100 & 0 & 0 & 0 & 0 & 0 & 0 & 0 & 0 \\
\hline $\mathrm{Cj} 1$ & 0 & 0 & 100 & 0 & 0 & 0 & 0 & 0 & 0 & 0 \\
\hline $\mathrm{Cj} 2$ & 0 & 0 & 0 & 100 & 0 & 0.25 & 0 & 0 & 0 & 0 \\
\hline Co1 & 0 & 0 & 0 & 0 & 100 & 0 & 0 & 0 & 0 & 0 \\
\hline $\mathrm{Co} 2$ & 0 & 0 & 0 & 0 & 0 & 98.85 & 0 & 0 & 0 & 0 \\
\hline Mo & 0 & 0 & 0 & 0 & 0 & 0 & 100 & 0 & 0 & 0 \\
\hline $\mathrm{Pt}$ & 0 & 0 & 0 & 0 & 0 & 0 & 0 & 99.90 & 0 & 0 \\
\hline $\mathrm{Tn}$ & 0 & 0 & 0 & 0 & 0 & 0 & 0 & 0 & 100 & 0 \\
\hline $\mathrm{Zs}$ & 0.60 & 0 & 0 & 0 & 0 & 0 & 0 & 0 & 0 & 95.70 \\
\hline
\end{tabular}

contrast to $\mathrm{Zs}$, a representative ring-porous hardwood with a single row of large vessels in the pore zone and a tangential arrangement of latewood vessels. This difference in the distribution of the vessels enabled clear discrimination with a resolution of $0.05 \mathrm{~mm}$. In fact, the CT images of Cc with a resolution of $0.05 \mathrm{~mm}$ exhibited local gray-level variations corresponding to the vessels, whereas those of Zs exhibited little variation in the earlywood because of the densely arranged vessels in the pore zone (Fig. 2, Cc, Zs). With a reduction in the resolution to $0.1 \mathrm{~mm}$; however, such a difference was hardly detected, and the regions of earlywood in $\mathrm{Cc}$ also exhibited dark colors with little variation, which made it similar to Zs.

To eliminate these errors, we have to revise each step in the system. In terms of the image dataset or the pretreatment of images, calibration of the gray levels to the actual density of wood seems to be a quite efficient and reliable approach because the specific gravities of the wood samples are actually quite different; in particular, $\mathrm{Pt}$ is known as a significantly light wood. Regarding feature extraction, the use of a multifeature extractor by combining the GLCM analysis with other filtering and transforms seems promising to improve the recognition accuracy. For example, feature extraction methods that focused on the periodicity of the images, such as the FFT and Gabor filter, would be effective for recognizing the differences between $\mathrm{Cc}$ and $\mathrm{Zs}$ because Zs has a clear periodicity in the radial direction derived from the tangential arrangement of the latewood vessels compared to $\mathrm{Cc}$. The periodicity in the radial direction derived from the ray may also be possibly detected and contribute to the improvement in the system.

\section{Conclusion}

We constructed a novel system for wood identification from low-resolution CT data using the GLCM and $k$-NN algorithm as a feature extractor and classifier, respectively. The system recognized 10 wood samples almost perfectly, although it is necessary to continue improvement. The current system is still at the level of individual recognition because the data were obtained from one wood block for each species; thus, data from multiple individuals should be added to the database to advance to the level of species recognition. In addition, the applicability of the system to various practical situations should be tested further; 
artifacts such as lacquerware and masks, which are covered by coating materials, are expected to be difficult because of the strong scattering from the metals in the coatings.

So far, the ability to recognize a wood species and to understand its specificities has been a task only accessible for specialists, more precisely, experienced anatomists. However, image recognition has the potential to offer the opportunity to introduce such knowledge to nonspecialists. Moreover, the GLCM effectively extracted the features of each wood species, indicating that the textural features may offer alternative keys to describe species specificities linked to still undefined anatomical features.

Acknowledgments The authors thank Mr. Ken'ichiro Yano, a sculptor, for providing the wood samples and valuable discussion in the early stage of this project. The authors also appreciate Ms. Izumi Kanai and Mr. Hajime Sorimachi for their enthusiasm and continuous technical support. This study was inspired by the pioneering master's thesis by Mr. Maekawa, Graduate School of Informatics, Kyoto University. This study was fully supported by Grants-in-Aid for Scientific Research (Grant Numbers 25252033 and 22300309) from the Japan Society for the Promotion of Science.

Open Access This article is distributed under the terms of the Creative Commons Attribution 4.0 International License (http://crea tivecommons.org/licenses/by/4.0/), which permits unrestricted use, distribution, and reproduction in any medium, provided you give appropriate credit to the original author(s) and the source, provide a link to the Creative Commons license, and indicate if changes were made.

\section{References}

1. IAWA Committee (1989) IAWA list of microscopic features for hardwood identification. Wheeler EA, Baas P, Gasson PE (eds) IAWA Bulletin (N.S.) 10:219-332

2. IAWA Committee (2004) IAWA list of microscopic features for softwood identification. Richter HG, Grosser D, Heinz I, Gasson P (eds) IAWA Bulletin (N.S.) 1:1-70

3. Schimleck LR, Michell AJ, Vinden P (1966) Eucalypt wood classification by NIR spectroscopy and principal components analysis. Appita J 49:319-324

4. Watanabe K, Abe H, Kataoka Y, Noshiro S (2011) Species separation of aging and degraded solid wood using near infrared spectroscopy. Jpn J Histor Bot 19:117-124

5. Horikawa Y, Mizuno-Tazuru S, Sugiyama J (2015) Near-infrared spectroscopy as a potential method for identification of anatomically similar Japanese diploxylons. J Wood Sci 61:251-261

6. Mizuno S, Torizu R, Sugiyama J (2010) Wood identification of a wooden mask using synchrotron X-ray micro-tomography. J Archaeol Sci 37:2842-2845

7. Khalid M, Lee ELY, Yusof R, Nadaraj M (2008) Design of an intelligent wood species recognition system. Int J Simul Sys Sci Technol 9:9-19

8. Bremananth R, Nithya B, Saipriya R (2009) Wood species recognition using GLCM and correlation. In: Proceedings of the 3rd International Conference on Advances in Recent Technologies in Communication and Computing, Kottayam, Kerala, India, pp 615-619. doi:10.1109/ArTCom.2009.10

9. Filho PLP, Oliveria LS, Britto AS Jr, Sabourin R (2010) Forest species recognition using color-based features. In: 20th International Conference on Pattern Recognition (ICPR), Istanbul, Turkey, pp 4178-4181. doi:10.1109/ICPR.2010.1015

10. Hermanson JC, Wiiedenhoeft AC (2011) A brief review of machine vision in the context of automated wood identification systems. IAWA J 32(2):233-250

11. Pan S, Kudo M (2011) Segmentation of pores in wood microscopic images based on mathematical morphology with a variable structuring element. Comput Electron Agric 75:250-260

12. Yusof R, Rosli NR, Khalid M (2010) Using Gabor filters as image multiplier for tropical wood species recognition system. In: 12th International Conference on Computer Modelling and Simulation (UKSim), Cambridge, United Kingdom, pp 289-294

13. Khalid M, Yusof R, Khairuddin ASM (2011) Tropical wood species recognition system based on multi-feature extractors and classifiers. In: Proceedings of 2011 2nd International Conference on Instrumentation Control and Automation (ICA), Bandung, Indonesia, pp 6-11

14. Martins J, Oliveira LS, Nisgoski S, Sabourin R (2013) A database for automatic classification of forest species. Machine Vision Appl 24:567-578

15. Wang HJ, Zhang GQ, Qi HN (2013) Wood recognition using image texture features. PLoS One 8(10):e76101. doi:10.1371/ journal.pone.0076101

16. Yuliastuti E, Suprijanto, Sasi SR (2013) Compact computer vision system for tropical wood species recognition based on pores and concentric curve. In: Proceedings of 2013 3rd International Conference on Instrumentation Control and Automation (ICA), Bali, Indonesia, pp 198-202

17. Yusof R, Khalid M, Khairuddin ASM (2013) Application of kernel-genetic algorithm as nonlinear feature selection in tropical wood species recognition system. Comput Electron Agric 93:68-77

18. Yadav AR, Anand RS, Dewal ML, Gupta S (2014) Analysis and classification of hardwood species based on Coiflet DWT feature extraction and WEKA workbench. In: International Conference on Signal Processing and Integrated Networks (SPIN), Noida, India, pp 9-13

19. Martins JG, Oliveira LS, Britto AS Jr, Sabourin F (2015) Forest species recognition based on dynamic classifier selection and dissimilarity feature vector representation. Machine Vision Appl 26:279-293

20. Haralick RM, Shanmugam K, Dinstein I (1973) Textural features for image classification. IEEE Trans Syst Man Cybern 3:610-621

21. R Core Team (2014) R: A language and environment for statistical computing. R Foundation for Statistical Computing, Vienna, Austria. http://www.R-project.org/. Accessed 7 Aug 2014

22. Bordese M, Alini W (2013) biOps: Image processing and analysis. R package version 0.2.2. http://CRAN.R-project.org/pack age=biOps. Accessed 7 Aug 2014

23. Albregtsen F (1995) Statistical texture measures computed from gray level cooccurrence matrices. In: Technical Note, Department of Informatics, University of Oslo, Norway

24. Venables WN, Ripley BD (2002) Modern applied statistics with S, 4th edn. Springer, New York 